\title{
Time-dependent transition of the immunoglobulin G subclass and immunoglobulin $E$ response in cancer patients vaccinated with cholesteryl pullulan-melanoma antigen gene-A4 nanogel
}

\author{
NORIAKI KYOGOKU ${ }^{1}$, HIROAKI IKEDA ${ }^{2}$, TAKAHIRO TSUCHIKAWA ${ }^{1}$, TAKEHIRO ABIKO ${ }^{1}$, AKI FUJIWARA ${ }^{1}$, \\ TAKEHIRO MAKI ${ }^{1}$, YOSHIYUKI YAMAMURA ${ }^{1}$, MASAOMI ICHINOKAWA ${ }^{1}$, KIMITAKA TANAKA ${ }^{1}$, \\ NAOKO IMAI $^{2}$, YOSHIHIRO MIYAHARA ${ }^{2}$, SHINICHI KAGEYAMA ${ }^{2}$, HIROSHI SHIKU $^{2}$ and SATOSHI HIRANO ${ }^{1}$ \\ ${ }^{1}$ Department of Gastroenterological Surgery II, Division of Surgery, Hokkaido University Graduate School of Medicine, \\ Sapporo, Hokkaido 060-8638; ${ }^{2}$ Department of Immuno-Gene Therapy, Mie University Graduate School of Medicine, \\ Tsu, Mie 514-8507, Japan
}

Received May 31, 2015; Accepted September 29, 2016

DOI: $10.3892 / 01.2016 .5253$

\begin{abstract}
A phase I+II clinical trial of vaccination with MAGE-A4 protein complexed with cholesteryl pullulan melanoma antigen gene-A4 nanogel (CHP-MAGE-A4) is currently underway in patients with MAGE-A4-expressing cancer. In the present study, the primary phase I endpoint was to test the safety of the administration of $300 \mu \mathrm{g}$ CHP-MAGE-A4 with and without OK-432. Another aim of the study was to clarify the details of the specific humoral immune response to vaccination. The 9 patients enrolled for phase I were vaccinated 6 times, once every 2 weeks: 3 patients with $100 \mu \mathrm{g}$ and 3 patients with $300 \mu \mathrm{g}$ CHP-MAGE-A4, and 3 patients with $300 \mu \mathrm{g}$ CHP-MAGE-A4 plus 0.5 clinical units of OK- 432 . Toxicities were assessed using Common Terminology Criteria for Adverse Events v3.0. Clinical response was evaluated by modified Response Evaluation Criteria in Solid Tumours. Immunological monitoring of anti-MAGE-A4-specific antibodies was performed by ELISA of pre- and post-vaccination patient sera. The 6 vaccinations produced no severe adverse events. Stable disease was assessed in 4/9 patients. Anti-MAGE-A4 total immunoglobulin (Ig)G titers increased in $7 / 9$ patients. Efficacious anti-MAGE-A4 IgG1, 2 and 3 antibody responses were observed in $7 / 9$ patients. Among them, positive conversions to T helper 2 (Th2)-type antibody responses (IgG4 and IgE) were observed after frequent vaccination in $4 / 7$ patients. The Th2 conversion was possibly associated with undesirable clinical observations, including
\end{abstract}

Correspondence to: Dr Noriaki Kyogoku, Department of Gastroenterological Surgery II, Division of Surgery, Hokkaido University Graduate School of Medicine, North 15, West 7, Kita-ku, Sapporo, Hokkaido 060-8638, Japan

E-mail: bigmuff118@yahoo.co.jp

Key words: immunoglobulin E, MAGE-A4, vaccine, antibody response, immunoglobulin $\mathrm{G}$ subclass progressive disease and the appearance of a new relapse lesion. The present study suggested that frequent vaccinations activated a Th2-dominant status in the cancer patients. The identification of a time-dependent $\operatorname{IgG}$ subclass and $\operatorname{IgE}$ antibody production during vaccination protocols may be a useful surrogate marker indicating a potentially undesirable change of the immunological environment for an effective antitumor immune response in cancer patients.

\section{Introduction}

Cancer vaccines show promise as novel cancer immunotherapies. A number of clinical trials of cancer vaccines have been conducted $(1,2)$, and safety (without severe adverse events) and efficacy in certain cases of advanced disease were demonstrated. However, marked clinical responses are rare and the expected effect has not been observed in a number of clinical trials $(1,2)$. To improve the efficacy of cancer vaccines, it is important to understand the precise immunological mechanisms that mediate the overall immune response of vaccinated patients.

Although the T helper (Th)1/Th2 balance in the antitumor immune response has been appreciated as an important factor to mediate the eradication of tumors, to the best of our knowledge, there have been few studies of serial assessment of the immunoglobulin $(\mathrm{Ig}) \mathrm{G}$ subclass and $\mathrm{IgE}$ response during cancer vaccine trials (3-6). However, it has been reported that IgG4 subclass antibodies impair antitumor immunity in melanoma patients (7).

A phase I+II clinical vaccine trial is currently being conducted using a complex of melanoma antigen gene-A4 (MAGE-A4) protein and cholesteryl pullulan (CHP) in patients with MAGE-A4-expressing cancer. MAGE-A4 antigen was first identified in the process of cloning cytotoxic $\mathrm{T}$ lymphocyte epitopes of melanoma cells (8), and it also belongs to the family of cancer testis antigens (9). As MAGE-A4 has reportedly been expressed in various cancer types, including uterine papillary serous carcinoma, ovarian cancer, lung cancer, melanoma, colorectal cancer, hepatocellular cancer and esophageal 
cancer (10-18), it is considered a potentially useful target antigen. Complexes of CHP nanoparticles that contain a tumor antigen are being used as a novel type of cancer vaccine, with a novel antigen delivery system for the MHC class I and II pathways $(6,7,19,20)$.

In the current trial, complexed recombinant MAGE-A4 protein and CHP nanogel (CHP-MAGE-A4) was administered to patients with unresectable tumors. The first 3 patients were treated with $100 \mu \mathrm{g}$ CHP-MAGE-A4, another 3 patients with $300 \mu \mathrm{g}$ CHP-MAGE-A4, and another 3 patients with $300 \mu \mathrm{g}$ CHP-MAGE-A4 plus OK-432, a compound that has been reported to stimulate toll-like receptor-4 and to activate antigen-presenting cells $(21,22)$. The primary phase I endpoint of this trial was to demonstrate the safety of administrating $300 \mu \mathrm{g}$ CHP-MAGE-A4 with and without OK-432. Another aim was to clarify additional immunological factors, particularly details of the humoral immune response, in patients treated with CHP-MAGE-A4. The present study reports the phase I results of this clinical trial and details of a time-dependent transition of the $\operatorname{IgG}$ subclass and $\operatorname{IgE}$ induction by CHP-MAGE-A4 vaccination. To the best of our knowledge, this is the first report to include these details of the immune response during cancer vaccination.

\section{Materials and methods}

Study design and treatment protocol. A phase I+II clinical trial of CHP-MAGE-A4 vaccine was designed to evaluate safety, immune response and clinical response. The primary endpoints were to evaluate the safety and optimal dose of the vaccine. The secondary endpoints were to investigate the immunological and clinical responses. Toxicities caused by the vaccination therapy were assessed using Common Terminology Criteria of Adverse Events (CTCAE) v3.0 (23). Immunological monitoring was performed using an enzyme-linked immunosorbent assay (ELISA) of patient sera obtained in the pre- and post-vaccination periods, as described below. To assess the clinical response, computed tomography (CT) imaging was performed prior to and following vaccination. Every measurable region was evaluated by the modified Response Evaluation Criteria in Solid Tumors (mRECIST) (24). The study protocol was approved by the Medical Ethics Committee of Hokkaido University (University Hospital Medical Information Network ID: 000001999; Sapporo, Hokkaido, Japan). Prior to every vaccination, intradermal testing with a 10 -fold dilution of CHP-MAGE-A4 was performed to confirm the absence of an immediate-type skin reaction. The CHP-MAGE-A4 vaccine was injected subcutaneously for a total of 6 cycles at 2-week intervals. To evaluate vaccine safety, a dose-escalation trial was conducted. Agents and applied doses were as follows: Group 1, $100 \mu \mathrm{g}$ MAGE-A4 recombinant protein complexed with $1.2 \mathrm{mg}$ CHP; group 2, $300 \mu \mathrm{g}$ MAGE-A4 recombinant protein complexed with $3.6 \mathrm{mg} \mathrm{CHP}$; and group 3, $300 \mu \mathrm{g}$ MAGE-A4 recombinant protein complexed with $3.6 \mathrm{mg}$ CHP and 0.5 clinical units of OK-432 (Chugai Pharmaceuticals, Tokyo, Japan) as an immune adjuvant. Group 1 patients were dosed first, followed by groups 2 and 3. Permission for dose escalation was granted when no adverse events greater than grade 3 had occurred in the preceding group during the 2 weeks following the second dose administration.
At 4 weeks after the last dose administration, the safety, immune response and clinical response were evaluated. Thereafter, additional vaccine was administered according to the patient's decision. Each of the 9 patients enrolled in the phase I part of this study received 6-25 immunizations.

Patient eligibility. Complete written informed consent was obtained from all patients at the time of enrollment. This study was performed in accordance with the guidelines of the Hokkaido University Institutional Review Board authorization. Eligibility criteria were as follows: i) Patients with locally-advanced, recurrent or metastatic tumors histologically confirmed as malignant and resistant to standard therapy; ii) patients with tumors expressing MAGE-A4 antigen, assessed by immunohistochemistry; iii) an Eastern Cooperative Oncology Group performance status of $0-2$ (25); iv) an age of $\geq 20$ years; v) $>4$ months survival expected; vi) adequate bone-marrow, cardiac, pulmonary, hepatic and renal functions, including the following: White blood cell count, $\geq 2,000 / \mu 1$; hemoglobin, $\geq 8.0 \mathrm{~g} / \mathrm{dl}$; platelets, $\geq 75,000 / \mu 1$; total bilirubin, $<1.5$ times the institutional normal upper limit (or, in patients with hepatic metastasis, $<3$ times); aspartate aminotransferase and alanine aminotransferase, $<2.5$ times the institutional normal upper limit (or, in patients with hepatic metastasis, $<5$ times); and creatinine, $<1.5$ times the institutional normal upper limit; and vii) the patient had no desire to become pregnant. Exclusion criteria were as follows: i) Positive for human immunodeficiency virus antibody; ii) multiple malignant diseases; iii) concurrent autoimmune disease; iv) a past history of anaphylaxis; v) active metastasis to the central nervous system; vi) concurrent anticancer therapy during the 4 weeks prior to the initiation of the trial (except with an anticancer drug that does not require drug holidays or hormone agents), including systemic steroids, immunosuppressive agents, irradiation or surgery for primary lesions; vii) pregnancy or breastfeeding; and viii) a decision by the principal investigator or physician in charge that the patient was unsuitable. The patient accrual began in August 2009 and ended in March 2013.

Preparation of CHP-MAGE-A4. Full length MAGE-A4 cDNA was cloned into a pET vector and introduced into Escherichia coli. Expression of His-MAGE-A4 protein was induced by the addition of isopropyl-L-thio- $\beta$-D-galactopyranoside to the bacterial cell culture; the produced protein was recovered and highly purified using a combination of chromatographic techniques, including metal chelating affinity, anion exchange, size exclusion and hydroxyapatite chromatography. CHP was synthesized by a chemical reaction between pullulan (mean molecular weight, $100 \mathrm{kDa}$ ) and cholesterol isocyanate in pyridine/dimethyl sulfoxide solution (Nippon Oil and Fat Co., Tokyo, Japan). Subsequent to purification by extraction and precipitation, the resultant CHP was emulsified in water and subsequently freeze-dried. When redissolved in water or buffers, CHP spontaneously forms nanoparticles. These nanoparticles $(20-50 \mathrm{~nm})$ contain the hydrophobic domains of cholesterol groups internally, which associate with the hydrophobic regions of the MAGE-A4 protein, forming a stable complex in solution. This complex of protein and CHP was 
used as the CHP-MAGE-A4 vaccine. These vaccines were kindly provided by the Department of Cancer Vaccine and Immuno-Gene Therapy of Mie University.

Detection of MAGE-A4 expression in tumors. To investigate MAGE-A4 antigen expression in tumors to determine whether patients could be enrolled in this study, each candidate's archival, formalin-fixed, paraffin-embedded tissue sections were subjected to immunohistochemical analysis. Patients with even slight MAGE-A4 positivity in the tumor were eligible for enrollment in this study. Immunohistochemical reactions were performed using the streptavidin-biotin-peroxidase method. The primary antibody used was MCV-1 $(2.8 \mathrm{mg} / \mathrm{ml}$; provided by Mie University), diluted 1:2,000 in Dako Antibody Diluent and Protein Block Serum-Free (Dako, Carpinteria, California, USA). MCV-1 is a monoclonal antibody (mAb) generated in mice immunized with human MAGE-A4 recombinant protein. Previous western blotting revealed that PC10, a human lung carcinoma cell line obtained from the Japanese Cancer Research Resources Bank (Tokyo, Japan), was positive for MAGE-A4 (data not shown); therefore, human testis tissue and PC10 were used as positive controls. The testis tissue specimen was obtained from an autopsy case with consent from the patient's family.

Archival tissue sections were deparaffinized in xylene and rehydrated in a graded series of ethanol solutions. Subsequent to washing in deionized water, antigens were unmasked by incubation for $7 \mathrm{~min}$ with citric acid buffer $(\mathrm{pH}$ 6.0) in a pressure cooker at $120^{\circ} \mathrm{C}$. After washing in deionized water, endogenous peroxidase activity was blocked by incubation for 5 min with $3 \%$ hydrogen peroxide in methanol at room temperature. After washing in deionized water and High-wash-phosphate buffered saline with Tween 20 (PBS-T; pH 7.7; $0.44 \mathrm{M} \mathrm{NaCl}, 0.1 \%$ Tween 20 in PBS; Wako Co. Ltd., Osaka, Japan), specimens were incubated overnight at $4^{\circ} \mathrm{C}$ with the aforementioned primary antibody. Following washing in High-wash-PBS-T, sections were incubated with peroxidase-labeled goat anti-mouse and -rabbit IgG (Fab') polyclonal antibody (catalog no. 41435; Histfine Simple Stain MAXPO [MULTI]; Nichirei, Tokyo, Japan) for $30 \mathrm{~min}$ at room temperature. After washing in High-wash-PBS-T, immunohistochemical reactions were visualized with freshly prepared 3,3'-diaminobenzidine tetrahydrochloride (Histofine SAB-PO [M] kit; Nichirei). Thereafter, slides were counterstained with hematoxylin and mounted on coverslips. Negative control staining was performed with 1:20 dilution mouse isotype IgG1 and IgG2a in Dako Antibody Diluent and Protein Block Serum-Free (Dako Japan, Co., Ltd., Tokyo, Japan).

If stained cells were microscopically observed, the specimen was labeled as 'positive,' regardless of the degree of positivity, as assessed by the determination of positive ratios for each localization (nucleus, cytoplasm, and nucleus and cytoplasm), by counting the most abundant 5 fields; the ratio of negative cells was also determined.

Detection of HLA class I antigen expression in tumors. To investigate the correlation between human leukocyte antigen (HLA) class I expression in tumors and clinical response, archival, formalin-fixed, paraffin-embedded tissue sections of each subject were analyzed by immunohistochemistry. Reactions were performed using the streptavidin-biotin-peroxidase method. The primary antibody was EMR8-5 mAb (catalog no. AB-46; 1:2,000 dilution; Hokudo, Sapporo, Japan), in a 3:1 solution of Dako Antibody Diluent and Protein Block Serum-Free (Dako Japan, Co., Ltd.) and 10\% normal goat serum (Histofine SAB-PO [R] kit; Nichirei). Previous western blotting with EMR8-5 revealed that PC10 was positive, and LCD was weakly positive, for HLA class I (data not shown). Thus, PC10 was used as a positive control, and human testis tissue as a negative control, for HLA class I immunohistochemistry. As an index of weak HLA class I expression, LCD, a human lung carcinoma cell line obtained from the Japanese Cancer Research Resources Bank, was used. Immunohistochemistry was performed as described above.

The intensity of the expression of HLA class I antigens was evaluated by comparing normal epithelial cells with tumor cells and was classified into the following grades: Staining intensity denser in tumor cells than normal epithelial cells $(+++)$, staining intensity equal in tumor and normal epithelial cells $(++)$, staining intensity fainter in tumor than normal epithelial cells (+), and tumor cells not stained (-). Endothelial cells were used in place of normal epithelial cells when sections of normal epithelial cells could not be obtained.

The positivity ratio for each grade was determined by area measurement using the image processing software ImageJ (http://rsb.info.nih.gov/ij/), as developed by the National Institutes of Health (Bethesda, MD, USA).

Evaluation of clinical responses. Toxicity was evaluated according to CTCAE v3.0. Tumor responses were assessed according to mRECIST. All known sites of disease were evaluated by CT scan after the 3rd vaccination cycle, and 4 weeks after the 6th cycle. mRECIST differs from existing RECIST in that all evaluable lesions with diameters $>10 \mathrm{~mm}$ are treated as target lesions. New lesions appearing after vaccination and initial non-target lesions growing to $>10 \mathrm{~mm}$ are treated as target lesions. The simple appearance of a new lesion is not treated as progressive disease (PD).

Serum samples. To analyze antigen-specific antibody responses, sera were collected prior to the initial vaccination and 2 weeks after each subsequent vaccination. All sera were stored at $-80^{\circ} \mathrm{C}$ until analyzed.

Detection of antibody responses to the MAGE-A4 protein. Specific antibodies in the sera were measured by ELISA. The MAGE-A4 recombinant protein in PBS was adsorbed onto immunoplates (442404; Nunc, Roskilde, Denmark) at a concentration of $20 \mathrm{ng} / 50 \mu \mathrm{l} /$ well overnight at $4^{\circ} \mathrm{C}$. Plates were washed in PBS with $0.05 \%$ Tween 20 (Sigma-Aldrich; Merck Millipore, Darmstadt, Germany) and then blocked for $2 \mathrm{~h}$ at room temperature with $200 \mu \mathrm{l} /$ well of $1 \%$ bovine serum albumin (BSA)/PBS. Serum samples were diluted in $1 \%$ BSA/PBS from 1:400 to 1:102,400. After washing, $100 \mu 1$ serum/well was added as the primary antibody and incubated overnight at $4^{\circ} \mathrm{C}$. Subsequent to washing, $100 \mu \mathrm{l} /$ well 
of 1:4,000 goat anti-human $\operatorname{IgG}(\mathrm{H}+\mathrm{L}$ chain)-horseradish peroxidase (HRP; MBL, Nagoya, Japan) in 1\% BSA/PBS was added as the secondary antibody and incubated for $5 \mathrm{~h}$ at $4^{\circ} \mathrm{C}$. Plates were washed, incubated with $100 \mu \mathrm{l} /$ well of TMB Substrate (Pierce; Thermo Fisher Scientific Inc., Waltham, MA, USA) for $3 \mathrm{~min}$ at room temperature. After that, $100 \mu \mathrm{l} /$ well of $0.18 \mathrm{M} \mathrm{H}_{2} \mathrm{SO}_{4}$ was added and the optical density (OD) of the sample was immediately read in a Microplate Spectrophotometer (SPECTRA MAX 190; Molecular Devices, Sunnyvale, CA, USA). The cutoff $\mathrm{OD}_{450}$ absorption value was calculated according to the following equation: The mean OD value of a 1:400 pooled serum sample from healthy donors $(n=24)$ plus $1.645 x$ standard deviation; the cutoff value determined was 0.288 . Similarly, healthy donor sera were used as controls to correct errors in every examination, and patients' OD values were corrected using the resultant calculated error ratios.

A positive reaction in seronegative patients was defined as follows; the OD of a 1:400 serum sample that exceeded the aforementioned cutoff value. A positive reaction in seropositive patients was defined as follows; the OD value of a 1:400 diluted serum sample after vaccinations that was further elevated than it had been prior to vaccinations.

Detection of IgG subclass antibody responses to the MAGE-A4 protein. Specific IgG subclass antibodies in the sera were measured by ELISA, as aforementioned, but using $100 \mu \mathrm{l} /$ well of diluted sheep anti-human $\operatorname{IgG1}, 2,3$, or 4 (H+L chain)-HRP (The Binding Site, Birmingham, UK) in $1 \%$ BSA/PBS as the secondary antibody, and incubations were for $5 \mathrm{~h}$ at $4^{\circ} \mathrm{C}$. Dilutions of each subclass were as follows: $\operatorname{IgG1} 1$ 1:25600; IgG2, 1:12800; IgG3, 1:12800; and IgG4, 1:12800. No IgG subclass antibody cross-reacts when used in these dilution ratios. The $\mathrm{OD}_{450}$ absorption cutoff value for each was calculated according to the following equation: The mean OD value of a 1:100 pooled serum sample from healthy donors $(n=24)$ plus $1.645 x$ standard deviation. Cutoff values of each IgG subclass were: IgG1, 0.192; IgG2, 0.140; IgG3, 0.076; and IgG4, 0.004. The percentage of the IgG4 fraction in the sera of the healthy donors was lowest. Furthermore, detection of anti-MAGE-A4-specific IgG4 antibody in the sera of healthy donors was difficult to obtain by ELISA. Therefore, even slight IgG4 responses following vaccination were considered to be positive.

Detection of IgE antibody responses to the MAGE-A4 protein. Specific IgE antibodies in the sera were measured by ELISA, as aforementioned, with differences in that as a primary antibody, the collected serum samples of each patient were diluted in 1\% BSA/PBS from 1:40 to 1:640. As a secondary antibody, $100 \mu \mathrm{l} /$ well of 1:1,000 rabbit polyclonal anti-human IgE (A0094; Dako Japan, Co., Ltd.) in 1\% BSA/PBS was added and incubated for $5 \mathrm{~h}$ at $4^{\circ} \mathrm{C}$. After washing, $100 \mu \mathrm{l} /$ well of 1:100 goat polyclonal anti-rabbit immunoglobulin or goat polyclonal anti-mouse immunoglobulin (as the negative control) (K1491; EnVision Kit-HRP, Dako Japan, Co., Ltd.) in 1\% BSA/PBS was added as a third antibody. Samples were incubated for $40 \mathrm{~min}$ at room temperature. After washing, OD was measured as aforementioned. There were non-specific reactions in the negative control wells, so these $\mathrm{OD}_{450}$ absorption values were deducted from all values obtained for sample wells.

The $\mathrm{OD}_{450}$ cutoff absorption value was calculated according to the following equation: The mean OD value of a 1:40 pooled serum sample from healthy donors $(n=24)$ plus $1.645 \mathrm{x}$ standard deviation; the cutoff value was determined to be 0.033 .

A positive reaction was defined as an OD value of a 1:40 serum sample exceeding the aforementioned cutoff value. There were no initially seropositive patients.

Statistical analysis. The $\chi^{2}$ test was used to assess the significance of the association of total $\mathrm{IgG}$ level with patient clinical response. In all tests, statistical significance was set at $\mathrm{P}<0.05$. All analyses were performed using StatView statistical software (version 5.0; SAS Institute Inc., Cary, NC, USA).

\section{Results}

Patient characteristics. For the phase I part of this study, 9 patients with the following cancer types were enrolled: 5 colon, 1 esophageal, 1 papilla of Vater, 1 breast and 1 pancreatic cancer case. Prior to the vaccinations, all patients had received standard therapy for refractory advanced, metastatic or recurrent cancer (Table I).

Expression of MAGE-A4 and HLA class I antigens in tumors. Staining regions varied from patient to patient, but there was a trend toward prominent cytoplasmic staining in the detection of MAGE-A4 (Table I). Immunohistochemical staining intensity varied from patient to patient in the detection of HLA class I. All 9 patients exhibited a certain degree of HLA class I antigen expression in the tumors (Table I). Representative sections after immunohistochemical staining are shown in Fig. 1.

Adverse events. Adverse events were assessed using CTCAE v3.0. All patients developed grade 1 local erythema at the injection sites, which resolved without any treatment. No induration or increase in reaction intensity was observed during sequential vaccinations. Grade 2 blister formation was observed at the distal portion of the injection site in patient 5 after the third vaccination. This symptom improved with non-steroid anti-inflammatory drug treatment, resolved after 24 days and was not observed during sequential vaccinations. No other adverse event or delayed-type hypersensitivity reaction associated with drug administration was observed in any patient (Table II).

Clinical responses. Tumor responses were assessed according to mRECIST criteria; 4 patients (patients 1, 4, 6, and 9) were assessed as exhibiting stable disease (SD), and the other 5 as PD. The clinical course of patient 4, who achieved the longest survival, is shown in Fig. 2. Prolonged $\mathrm{SD}$, of $\sim 20$ weeks, was maintained by vaccination in this patient diagnosed with frequently relapsing colon cancer. The size of the mediastinal relapse tumor in this patient had not changed for $>12$ months and necrosis had increased. However, 5 months after the first vaccination, new and/or 


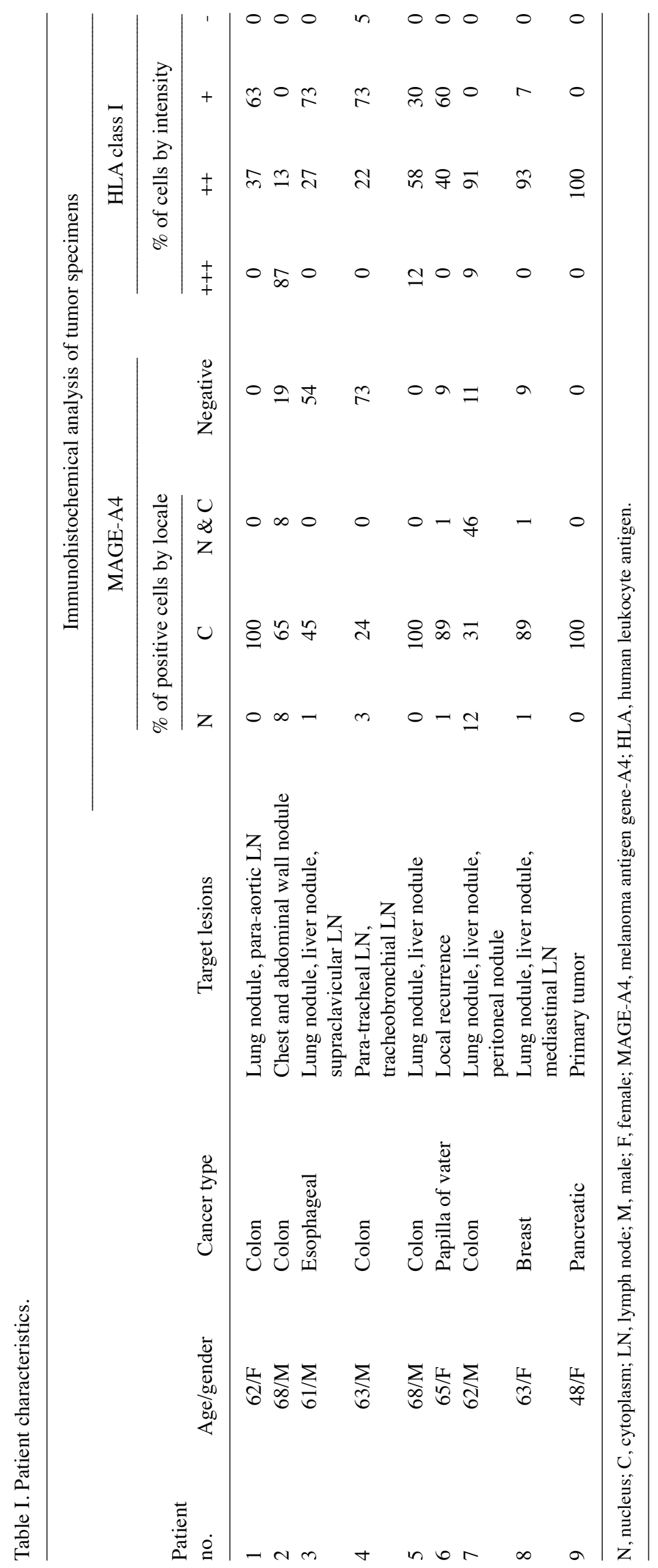




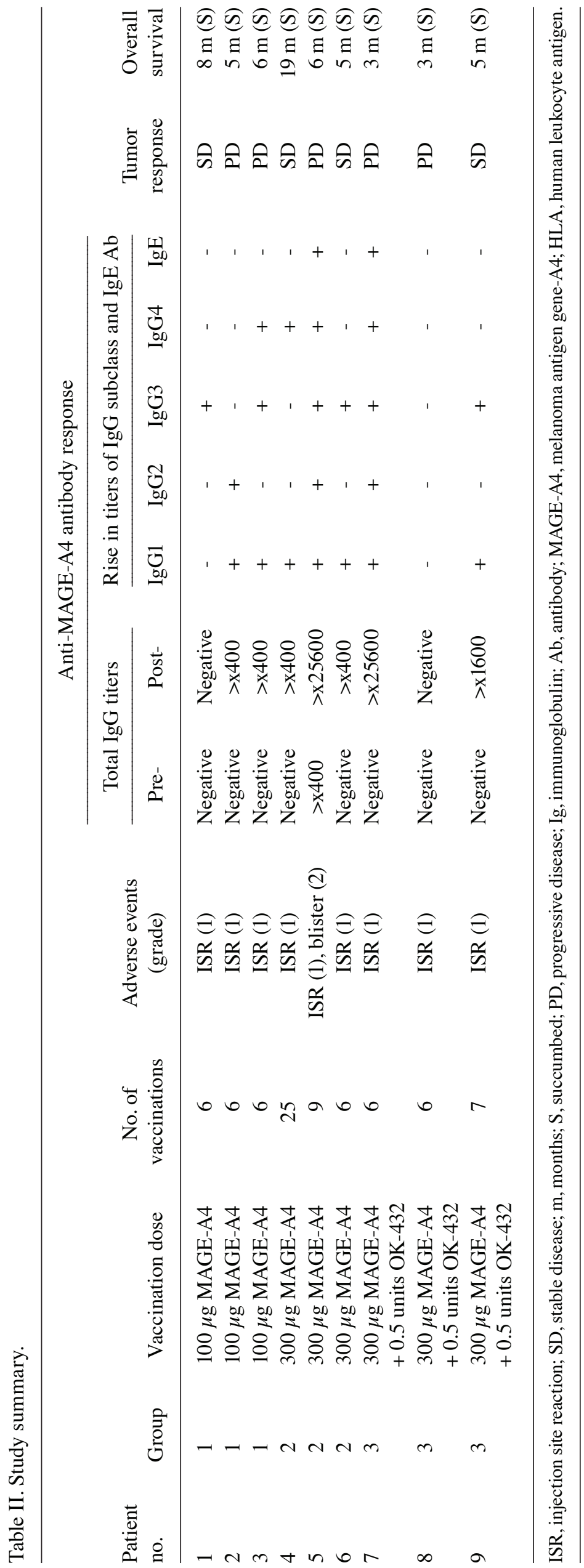



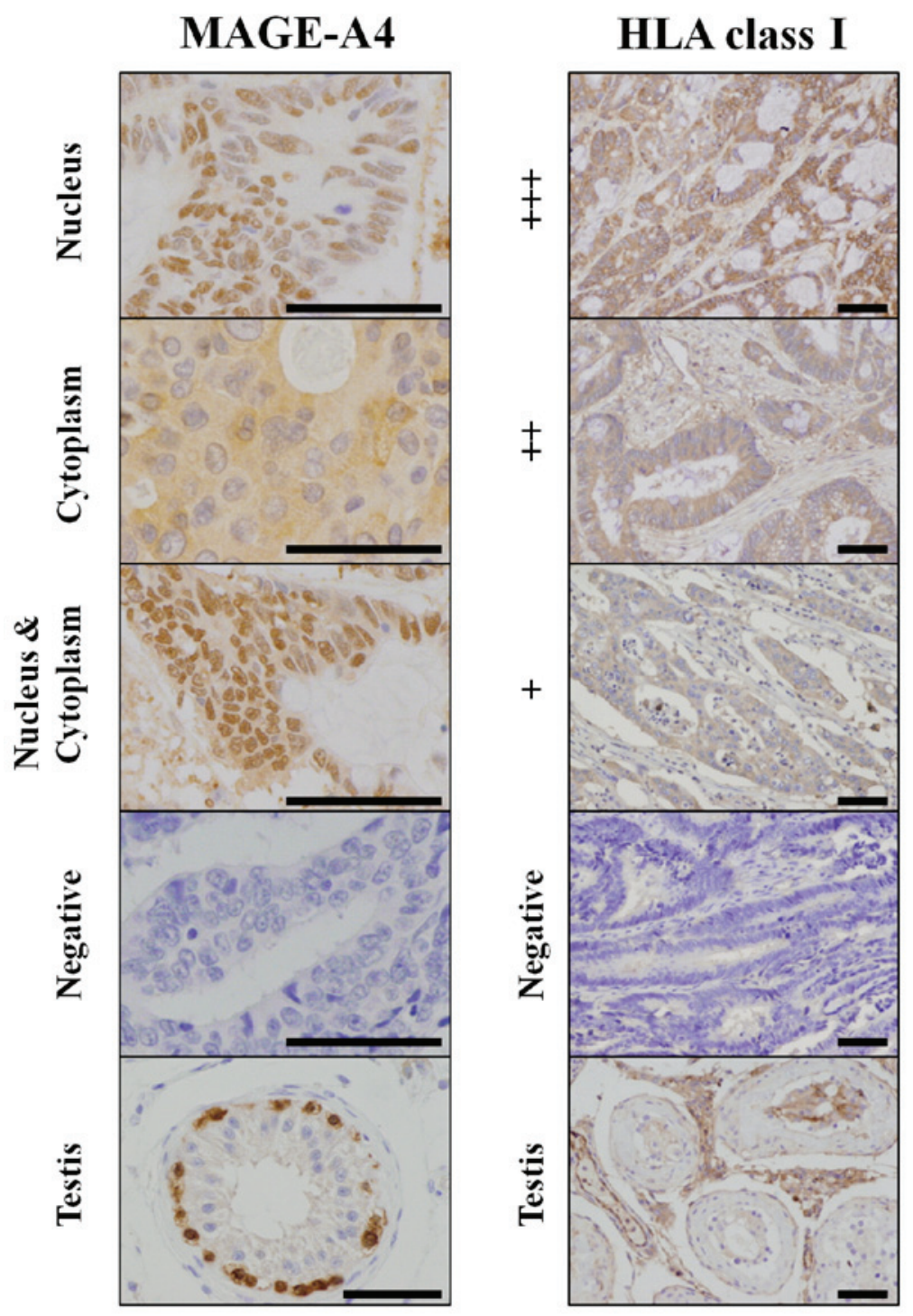

Figure 1. MAGE-A4 and HLA class I expression in cancer and control tissues. Immunohistochemical staining regions of MAGE-A4 and staining intensity of HLA class I varied from patient to patient. Three types of localization patterns of MAGE-A4 (nucleus, cytoplasm, and nucleus and cytoplasm) were observed. Staining intensity of HLA class I was classified into 3 grades by comparing normal epithelial cells with tumor cells. Human testis tissue was used as a positive control for MAGE-A4 immunohistochemistry, and as a negative control in the detection for HLA class I immunohistochemistry. Scale bar, 10 $\mu \mathrm{m}$. MAGE-A4, melanoma antigen gene-A4; HLA, human leukocyte antigen.

enlarged lesions were detected. No tumor regression was observed in any patient.

Antibody responses to the MAGE-A4 protein. Total $\mathrm{IgG}$ responses to the MAGE-A4 protein were determined by ELISA. As shown in Fig. 3 and Table II, 1 of the patients (patient 5) had pre-existing antibodies to MAGE-A4, and was considered seropositive. The other 8 patients had no antibodies to MAGE-A4 prior to vaccination, and were considered seronegative; 6 of these patients (patients 2, 3, 4, 6, 7 and 9) seroconverted after vaccinations. In the patient with pre-existing anti-MAGE-A4 antibody prior to vaccination, the antibody titer increased after the vaccinations. The anti-MAGE-A4 antibody level increased in 7 of the 9 patients ( 2 in group 1, 3 in group 2 and 2 in group 3). There was a trend toward an earlier and higher titer increase in the patients of groups 2 and 3 compared with those of group 1. No significant correlation (by $\chi^{2}$ analysis, $\mathrm{P}=0.858$ ) was observed between total $\operatorname{IgG}$ titer and clinical response (SD: Patients 1, 4, 6 and 9 vs. PD: Patients 2, 3, 5, 7 and 8).

IgG subclass- and IgE antibody responses to the MAGE-A4 protein. $\operatorname{IgG1}, 2$ and 3 antibody responses can be driven by Th1 and Th2 cells $(26,27)$. On the other hand, IgG4 and IgE antibody responses are mediated mainly by Th2 cells $(26,27)$. In all 7 patients who were considered positive for an antibody response to MAGE-A4 protein, the IgG1 antibody titer rose (Fig. 4). A trend toward increasing IgG3 antibody titers was observed after the increase of IgG1 antibody titers. Positive conversions to Th2-dominant antibody responses ( $\mathrm{IgG} 4$ and $\operatorname{IgE}$ ) were also observed after vaccination in 4 patients (patients 3, 4, 5 and 7), who had been positive for Th1-dominant antibody (IgG1, 2 and 3) responses. In the patients who had Th2 antibody responses, $1 \mathrm{SD}$ and $3 \mathrm{PD}$ clinical responses were observed, while patients without a Th2 antibody response exhibited $2 \mathrm{PD}$ and $3 \mathrm{SD}$ clinical responses. 


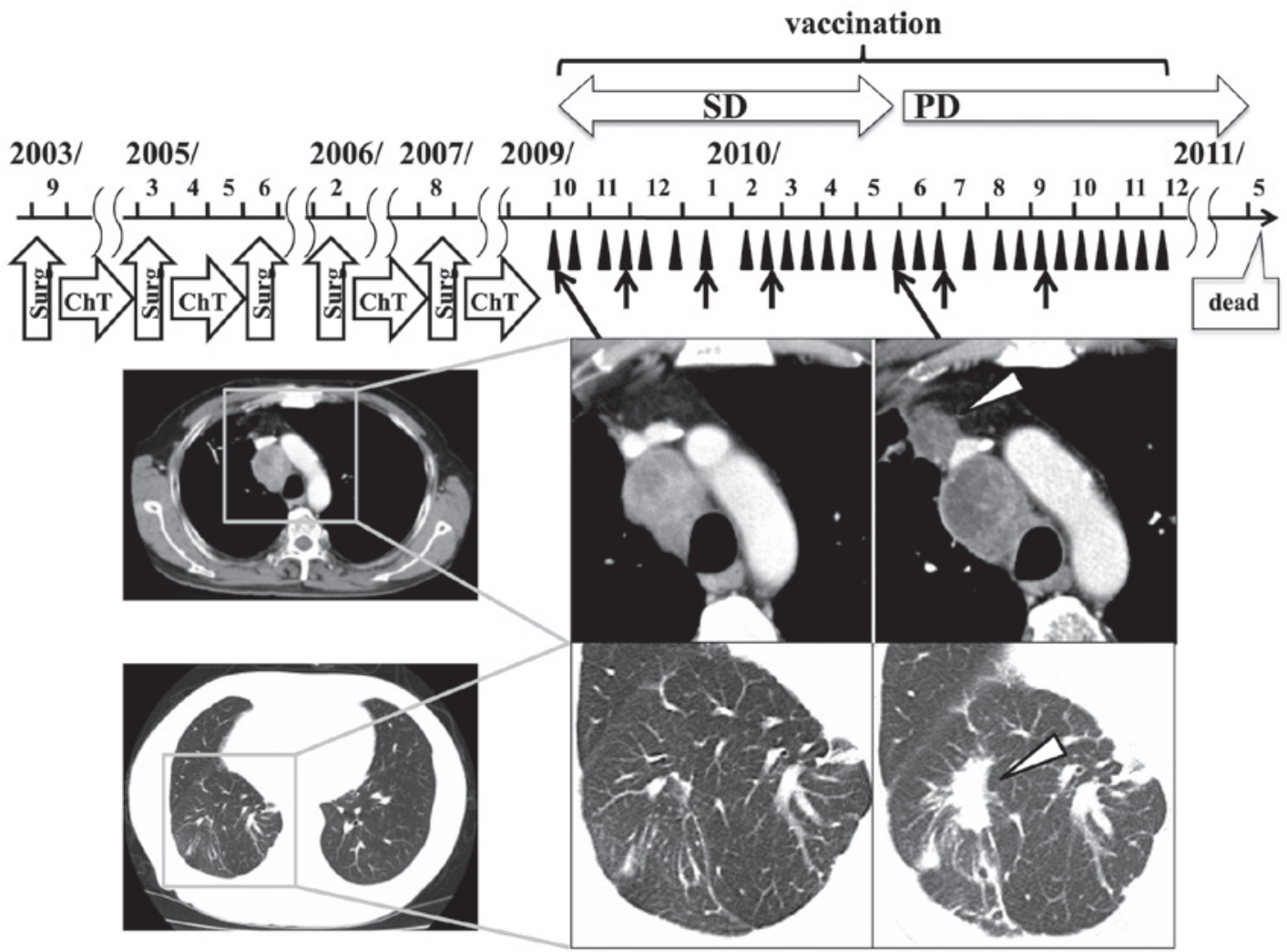

Figure 2. Clinical course of patient 4 . Prolonged SD of $\sim 20$ weeks was maintained by vaccination in a patient diagnosed with colon cancer that had relapsed frequently. Black arrows indicate CT. Black arrowheads indicate vaccination. White arrowheads indicate new and/or enlarged lesions. SD, stable disease; PD, progressive disease; Surg, surgery; ChT, chemotherapy.

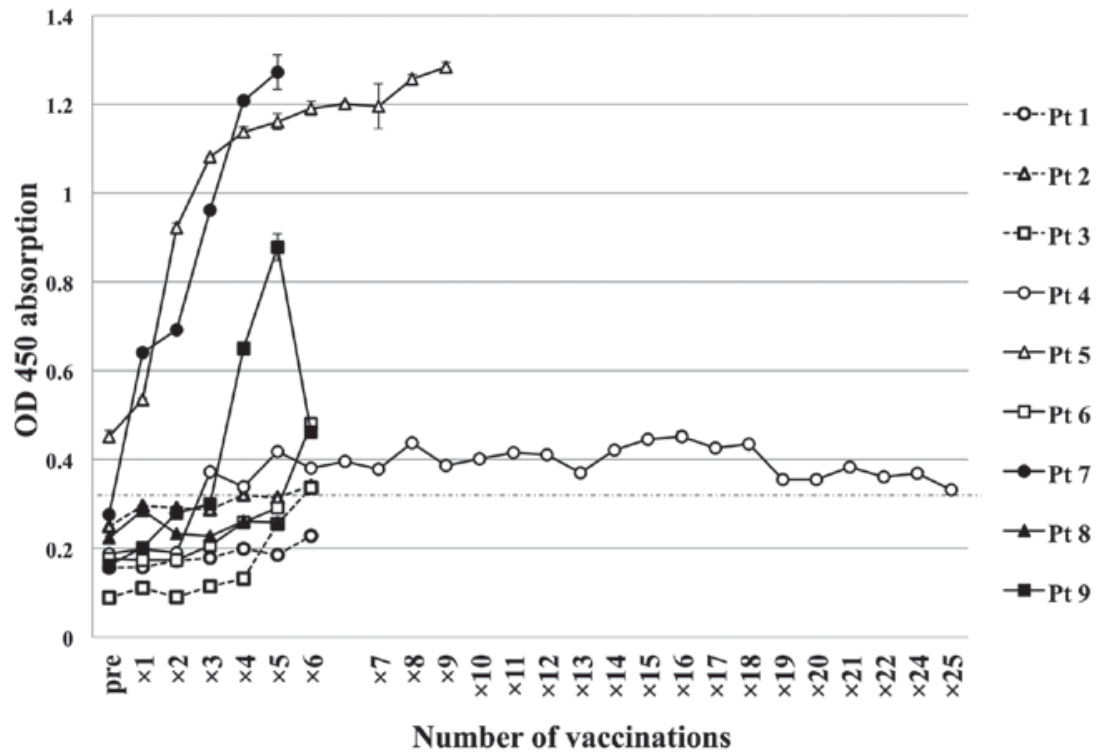

Figure 3. Antibody responses to the MAGE-A4 protein. Anti-MAGE-A4 antibody titer increased in 7 of 9 patients (2 patients in group 1, 3 patients in group 2 and 2 patients in group 3). A trend can be observed toward earlier and greater antibody titer increases in the patients of groups 2 and 3 compared with those of group 1. Pt, patient; OD, optical density; MAGE-A4, melanoma antigen gene-A4.

Total IgG4 antibody levels, including those not specific for MAGE-A4, were measured in the sera of all 9 patients prior to and following the vaccinations, but significant disparities were not found during the course of the study (data not shown). As the sample size was small and statistical analysis was difficult, trends in the results were assessed, but none were observed when comparing tumor suppressive effects and other factors. For example, the expression of MAGE-A4 in the tumor of patient 4 , who was the longest survivor, was only $24 \%$. Although the immune response of patient 4 may reflect the clinical response, other patients (patients 5,7 and 9), who had greater immune responses, did not have favorable clinical 

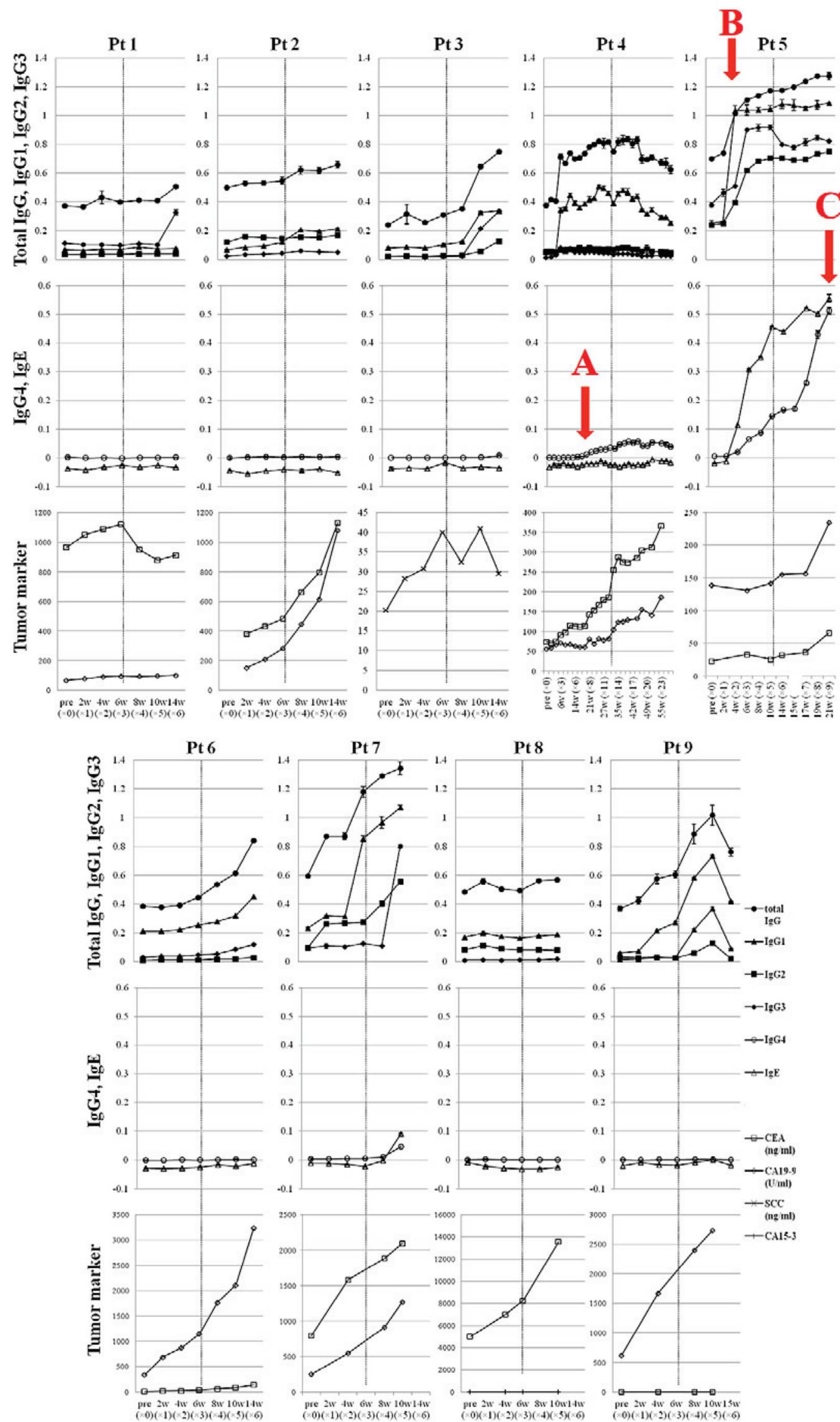

Figure 4. Melanoma antigen gene-A4-specific IgG1 antibody titer was elevated in 7 patients. Positive conversions to a Th2-dominant antibody response (IgG4 and/or IgE production) were observed after frequent vaccination in 4 patients (patient 3, 4,5 and 7) that had been positive for Th1-dominant antibody responses (IgG1, 2 and 3). Pathognomonic signs observed were as follows: A, patient 4 developed a new lesion, the tumor marker level began to rise and an IgG4 antibody response occurred at the same time; B, patient 5 developed a blister, evaluated as a grade 2 adverse event at the moment that the IgG1 antibody titer rose markedly; and C, patient 5 had an immediate-type skin reaction to the prevaccination intradermal test at the moment that the IgE antibody titer rose to the highest level. Ig, immunoglobulin; Th, T helper; w, weeks; Pt, patient; CEA, carcinoembryonic antigen; CA, cancer antigen; SCC, squamous cell carcinoma. 
responses (Fig. 4). On the other hand, among the 4 patients who had rising MAGE-A4-specific IgG4 or IgE, characteristic clinical events were observed in 2 of them. A new relapse lesion was observed simultaneously with the rise of MAGE-A4-specific IgG4 in patient 4 (Fig. 4A). In patient 5, a blister was observed at the moment that the $\operatorname{IgG1}$ antibody titer rose markedly (Fig. 4B), and an immediate-type skin reaction to the prevaccination intradermal test was observed when the $\operatorname{IgE}$ antibody titer rose to the maximal level, so it was decided that patient 5 should discontinue vaccinations (Fig. 4C).

\section{Discussion}

With respect to the safety of this CHP-MAGE-A4 vaccine, there were no adverse events greater than grade 3 at least until after the sixth vaccination. Only patient 5 had grade 2 blister formation in the present trial. A previous study detailed severe adverse events after peptide vaccination in a patient with highly boosted cellular and humoral responses (28). Therefore, there is a possibility that the marked rise in IgG1 antibody titer was associated with the grade 2 blister formation in patient 5 .

Cancer immunotherapy requires a relatively long period prior to the onset of tumor response, and the tumor could continue to grow for several weeks after the first vaccination (27). Therefore, mRECIST was used to precisely evaluate the efficacy of this therapy in the present study; 4 patients were assessed as SD and the other 5 as PD.

MAGE-A4-specific antibody responses were detected in 7 of the 9 patients in this study (group 1, 2/3; group 2, 3/3; group $3,2 / 3$ ). The observed trend was that the antibody titer rose earlier and higher in patients of groups 2 and 3 than in those of group 1. Therefore, the administration of $300 \mu \mathrm{g}$ CHP-MAGE-A4 could prove to be more effective than $100 \mu \mathrm{g}$ for eliciting a strong immune response. Although several studies suggested that stereotypical immunostimulation would have certain benefits, depending on the particular combination of vaccine agent and immune adjuvant, in the present study, the combination with OK-432 did not enhance the effect of CHP-MAGE-A4 vaccination. There was no association between total $\mathrm{IgG}$ response and tumor response. A possible reason proposed was that a favorable tumor response could not be detected when there were a number of tumors or if they were bulky. For example, although patients 5 and 7 had the highest MAGE-A4-specific antibody titers, they did not have any tumor responses and were evaluated as PD as they had also bulky metastatic tumors that filled a large portion of the entire liver (Table II).

The Th1/Th2 balance is closely associated with antitumor immunity (29). Therefore, the evaluation and control of the Th1/Th2 balance in patients treated with cancer vaccines are considered to be necessary for successful therapy. Generally, IgG1, 2 and 3 antibodies are induced in Th1 and Th2 cytokine environments (30,31). By contrast, IgG4 and IgE antibodies are induced in the Th2-dominant cytokine environment $(30,31)$. The intensity of effector function has been regarded as follows: $\mathrm{IgG} 1=\mathrm{IgG} 3 \gg \mathrm{IgG} 2=\mathrm{IgG} 4(30,31)$.

$\mathrm{IgE}$ is known as an antibody produced during allergic activity. IgG4 is known as an IgE-blocking antibody that is induced after frequent administration of allergen during hyposensitization therapy $(32,33)$. In studies of humoral immunity in patients with tumors, Th2-dominant cytokines have hardly attracted attention, while the Th1-dominant cytokine environment has been well investigated. There have been studies on the antitumor effects of $\operatorname{IgE}$ antibody $(26,27,34)$, but the precise immunological mechanisms involved remain unclear. Recently, Karagiannis et al reported that IgG4 subclass antibodies impair antitumor immunity in melanoma (7). So there is a focused negative effect induced by IgG4 on the antitumor immune response. There have been few studies regarding the $\mathrm{IgG}$ subclasses and $\mathrm{IgE}$ during cancer vaccination. To the best of our knowledge, the present study is the first to evaluate the time-dependent transition of the $\mathrm{IgG}$ subclass and $\operatorname{IgE}$ during cancer vaccination. In this study, the CHP-MAGE-A4 vaccine induced mainly the Th1-dominant antibody response of $\operatorname{IgG1}, 2$ and 3 production. However, positive conversions to the Th2-dominant antibody response meant that IgG4 and IgE were also observed after several rounds of vaccination in patients who previously had been positive for Th1-dominant antibody responses. In total, 3 PD and $1 \mathrm{SD}$ clinical responses were observed in patients who showed the Th 2 conversion in the antigen-specific antibody response, while there were $2 \mathrm{PD}$ and $3 \mathrm{SD}$ clinical responses in patients without Th2 conversion. These results suggest a possible association between the time-dependent Th 2 conversion and the clinical benefit to the patient, although this issue must be rigorously confirmed in later stages of clinical trials aiming to address clinical response in a stringent manner with larger enrollment.

Although it is unknown whether the reaction of the Th2-dominant antibody response depends on frequent medication or time after the first medication or superfluous Th1 reaction, in the present study, the rise in IgG4 antibody titer was delayed compared with the IgG1 response after frequent vaccination, confirming similar findings of a past study (5). IgG4 and IgE antibody responses were positive in patients 5 and 7, who had vigorous IgG1, 2 and 3 responses. These data suggest that a robust Th1-dominant antibody response may lead to conversion from a Th1 to a Th2 cytokine environment. By contrast, patient 4 , who was mildly positive for a Th1-dominant antibody response, had only an IgG4 antibody response, and prolonged survival. However, this patient developed a new lesion, rising levels of tumor marker and an IgG4 antibody response at the same time, suggesting that the IgG4 antibody response may be a sensitive surrogate marker of undesirable change in the antitumor immune response. The current data showed that several injections of cancer vaccine were safe, but may cause an allergic reaction that is undesirable for creation of cancer immunity due to the similarity to conditions created during hyposensitization therapy for allergies. In past studies, self-antigen-derived cancer vaccines elicited allergic reactions. Moreover, the allergic reaction resolved after elimination of specific amino acid sequences known to evoke an allergic reaction from studies of the peptide involved $(35,36)$. If characteristics of the IgG4 and IgE epitopes of MAGE-A4 were clarified, it would be possible to avoid an allergic-like reaction by the elimination of the pertinent IgG4 and IgE epitopes from the vaccine agent. 
In conclusion, the current results suggest that clinicians should be aware that frequent vaccine administration may induce a Th2 cytokine environment, and that there is a possibility that the $\operatorname{IgG}$ subclass and $\mathrm{IgE}$ antibody responses are useful as surrogate markers for an undesirable change in antitumor immunity, providing an indication to discontinue vaccine administration. Monitoring the time-dependent transitions of the IgG subclass and IgE levels will be important during cancer vaccination therapy. It may be necessary to reconsider protocols requiring frequent vaccinations at relatively short intervals. Patient sera from past cancer vaccine trials will aid in precisely addressing this possibility and also in clarifying the precise immunological mechanisms of the $\mathrm{Th} 2$ transition of the immune response induced by cancer vaccination.

\section{Acknowledgements}

The authors would like to thank Dr. Masaki Miyamoto (Department of Gastroenterological Surgery II, Division of Surgery, Hokkaido University Graduate School of Medicine) for great mentorship and contribution as a leader of the research group. The authors would also like to thank the Department of Cancer Vaccine and Immuno-Gene Therapy, Mie University, for providing the CHP-MAGE-A4 vaccine and MAGE-A4-specific antibody.

\section{References}

1. Rosenberg SA, Yang JC and Restifo NP: Cancer immunotherapy: Moving beyond current vaccines. Nat Med 10: 909-915, 2004.

2. Itoh K, Yamada A, Mine T and Noguchi M: Recent advances in cancer vaccines: An overview. Jpn J Clin Oncol 39: 73-80, 2009.

3. Mine T, Sato Y, Noguchi M, Sasatomi T, Gouhara R, Tsuda N, Tanaka S, Shomura H, Katagiri K, Rikimaru T, et al: Humoral responses to peptides correlate with overall survival in advanced cancer patients vaccinated with peptides based on pre-existing, peptide-specific cellular responses. Clin Cancer Res 10: 929-937, 2004.

4. Ullenhag GJ, Frödin JE, Strigård K, Mellstedt $\mathrm{H}$ and Magnusson CG: Induction of IgG subclass responses in colorectal carcinoma patients vaccinated with recombinant carcinoembryonic antigen. Cancer Res 62: 1364-1369, 2002.

5. Aoki M, Ueda S, Nishikawa H, Kitano S, HIrayama M, Ikeda H, Toyoda H, Tanaka K, Kanai M, Takabayashi A, et al: Antibody responses against NY-ESO-1 and HER2 antigens in patients vaccinated with combinations of cholesteryl pullulan (CHP)-NY-ESO-1 and CHP-HER2 with OK-432. Vaccine 27: 6854-6861, 2009.

6. Kageyama S, Kitano S,Hirayama M,Nagata Y,Imai H,Shiraishi T, Akiyoshi K, Scott AM, Murphy R, Hoffman EW, et al: Humoral immune responses in patients vaccinated with 1-146 HER2 protein complexed with cholesteryl pullulan nanogel. Cancer Sci 99: 601-607, 2008.

7. Karagiannis P, Gilbert AE, Josephs DH, Ali N, Dodev T, Saul L, Correa I, Roberts L, Beddowes E, Koers A, et al: IgG4 subclass antibodies impair antitumor immunity in melanoma. J Clin Invest 123: 1457-1474, 2013.

8. De Plaen E, Naerhuyzen B, De Smet C, Szikora JP and Boon T: Alternative promoters of gene MAGE4a. Genomics 40: 305-313, 1997.

9. Scanlan MJ, Gure AO, Jungbluth AA, Old LJ and Chen YT: Cancer/testis antigens: An expanding family of targets for cancer immunotherapy. Immuno Rev 188: 22-32, 2002.

10. Resnick MB, Sabo E, Kondratev S, Kerner H, Spagnoli GC and Yakirevich E: Cancer-testis antigen expression in uterine malignancies with an emphasis on carcinosarcomas and papillary serous carcinomas. Int J Cancer 101: 190-195, 2002.
11. Yakirevich E, Sabo E, Lavie O, Mazareb S, Spagnoli GC and Resnick MB: Expression of the MAGE-A4 and NY-ESO-1 cancer-testis antigens in serous ovarian neoplasms. Clin Can Res 9: 6453-6460, 2003.

12. Tajima K, Obata Y, Tamaki H, Tamaki H, Yoshida M, Chen YT, Scanlan MJ, Old LJ, Kuwano H, Takahashi T, et al: Expression of cancer/testis (CT) antigens in lung cancer. Lung Cancer 42: 23-33, 2003.

13. Prasad ML, Jungbluth AA, Petal SG, Iversen K, Hoshaw-Woodard S and Busam KJ: Expression and significance of cancer/testis antigens in primary mucosal melanoma of the head and neck. Head Neck 26: 1053-1057, 2004.

14. Li M, Yuan YH, Han Y, Liu YX, Yan L, Wang Y and Gu J: Expression profile of cancer-testis genes in 121 human colorectal cancer tissue and adjacent normal tissue. Clin Can Res 11: 1809-1814, 2005.

15. Gure AO, Chua R, Williamson B, Gonen M, Ferrera CA, Gnjatic S, Ritter G, Simpson AJ, Chen YT, Old LJ and Altorki NK: Cancer-testis genes are coordinately expressed and are markers of poor outcome in non-small cell lung cancer. Clin Can Res 11: 8055-8062, 2005.

16. Peng JR, Chen HS, Mou DC, Cao J, Cong X, Qin LL, Wei L, Leng XS, Wang Y and Chen WF: Expression of cancer/testis (CT) antigens in Chinese hepatocellular carcinoma and its correlation with clinical parameters. Cancer Lett 219: 223-232, 2005.

17. Barrow C, Browning J, MacGregor D, Davis ID, Sturrock S, Jungbluth AA and Cebon J: Tumor antigen expression in melanoma varies according to antigen and stage. Clin Can Res 12: 764-771, 2006.

18. Akcakanat A, Kanda T, Tanabe T, Komukai S, Yajima K, Nakagawa S, Ohashi M and Hatakeyama K: Heterogeneous expression of GAGE, NY-ESO-1, MAGE-A and SSX proteins in esophageal cancer: Implications for immunotherapy. Int $\mathrm{J}$ Cancer 118: 123-128, 2006

19. Kageyama S, Wada H, Muro K, Niwa Y, Ueda S, Miyata H, Takiguchi S, Sugino SH, Miyahara Y, Ikeda H, et al: Dose-dependent effects of NY-ESO-1 protein vaccine complexed with cholesteryl pullulan (CHP-NY-ESO-1) on immune responses and survival benefits of esophageal cancer patients. J Transl Med 11: 246, 2013.

20. Gu XG, Schmitt M, Hiasa A, Nagata Y, Ikeda H, Sasaki Y, Akiyoshi K, Sunamoto J, Nakamura H, Kuribayashi K and Shiku H: A novel hydrophobized polysaccharide/oncoprotein complex vaccine induces in vitro and in vivo cellular and humoral immune responses against HER2-expressing murine sarcomas. Cancer Res 58: 3385-3390, 1998.

21. Okamoto M, Furuichi S, Nishioka Y, Oshikawa T, Tano T, Ahmed SU, Takeda K, Akira S, Ryoma Y, Moriya Y, et al: Expression of toll-like receptor 4 on dendritic cells is significant for anticancer effect of dendritic cell-based immunotherapy in combination with an active component of OK-432, a streptococcal preparation. Cancer Res 64: 5461-5470, 2004.

22. Nakahara S, Tsunoda T, Baba T, Asabe S and Tahara H: Dendritic cells stimulated with a bacterial product, OK-432, efficiently induce cytotoxic $\mathrm{T}$ lymphocytes specific to tumor rejection peptide. Cancer Res 63: 4112-4118, 2003.

23. No authors listed: Japanese translation of common terminology criteria for adverse events (CTCAE), and instructions and guidelines. Int J Clin Oncol 9 (Suppl 3): 1-82, 2004 (In Japanese).

24. Wolchok JD, Hoos A, O'Day S, Weber JS, Hamid O, Lebbe C, Maio M, Binder M, Bohnsack O, Nichol G, et al: Guidelines for the evaluation of immune therapy activity in solid tumors: Immune-related response criteria. Clin Cancer Res 15: 7412-7420, 2009.

25. Oken MM, Creech RH, Tormey DC, Horton J, Davis TE, McFadden ET and Carbone PP: Toxicity and response criteria of the Eastern Cooperative Oncology Group. Am J Clin Oncol 5: 649-655, 1982.

26. Nigro EA, Brini AT, Soprana E, Ambrosi A, Dombrowicz D, Siccardi AG and Vangelista L: Antitumor IgE adjuvanticity: Key role of Fc epsilon RI. J Immunol 183: 4530-4536, 2009.

27. Reali E, Greiner JW, Corti A, Gould HJ, Bottazzoli F, Paganelli G, Schlom J and Siccardi AG: IgEs targeted on tumor cells: Therapeutic activity and potential in the design of tumor vaccines. Cancer Res 61: 5517-5522, 2001.

28. Yoshida K, Noguchi M, Mine T, Komatu N, Yutani S, Ueno T, Yanagimoto H, Kawano K, Itoh K and Yamada A: Characteristics of severe adverse events after peptide vaccination for advanced cancer patients: Analysis of 500 cases. Oncol Rep 25: 57-62, 2011. 
29. Kidd P: Th1/Th2 balance: The hypothesis, its limitations, and implications for health and disease. Altern Med Rev 8: 223-246, 2003.

30. Romagnani S: Th1 and Th2 in human diseases. Clin Immunol Immunopathol 80: 225-235, 1996.

31. Shaw DR, Khazaeli MB and LoBuglio AF: Mouse/human chimeric antibodies to a tumor-associated antigen: Biologic activity of the four human IgG subclasses. J Natl Cancer Inst 80 : $1553-1559,1988$

32. Jutel $\mathrm{M}$ and Akdis CA: Immunological mechanisms of allergen-specific immunotherapy. Allergy 66: 725-732, 2011.

33. Aalberse RC, Stapel SO, Schuurman J and Rispens T: Immunoglobulin G4: An odd antibody. Clin Exp Allergy 39: 469-477, 2009.
34. Riemer AB, Untersmayr E, Knittelfelder R, Duschl A, Pehamberger H, Zielinski CC, Scheiner O and Jensen-Jarolim E: Active induction of tumor-specific $\operatorname{IgE}$ antibodies by oral mimotope vaccination. Cancer Res 67: 3406-3411, 2007.

35. Yamada A, Yano H, Takao Y, Ono T, Mastumoto T and Itoh K: Nonmutated self-antigen-derived cancer vaccine peptides elicit an IgE-independent but mast cell-dependent immediate-type skin reaction without systemic anaphylaxis. J Immunol 176: 857-863, 2006.

36. Ohkouchi S, Yamada A, Imai N, Mine T, Harada K, Shichijo S, Maeda Y, Saijo Y, Nukiwa $\mathrm{T}$ and Itoh K: Non-mutated tumor-rejection antigen peptides elicit type-I allergy in the majority of healthy individuals. Tissue Antigens 59: 259-272, 2002. 